\title{
Fusing Simultaneous EEG and fMRI Using Functional and Anatomical Information
}

\author{
Hansen, Sofie Therese; Winkler, Irene; Hansen, Lars Kai; Müller, Klaus-Robert; Dahne, Sven
}

Published in:

Proceedings of the 2015 International Workshop on Pattern Recognition in Neurolmaging (PRNI 2015)

Link to article, DOI:

10.1109/PRNI.2015.22

Publication date:

2015

Document Version

Peer reviewed version

Link back to DTU Orbit

Citation (APA):

Hansen, S. T., Winkler, I., Hansen, L. K., Müller, K-R., \& Dahne, S. (2015). Fusing Simultaneous EEG and fMRI Using Functional and Anatomical Information. In Proceedings of the 2015 International Workshop on Pattern Recognition in Neurolmaging (PRNI 2015) (pp. 33-36). IEEE. https://doi.org/10.1109/PRNI.2015.22

\section{General rights}

Copyright and moral rights for the publications made accessible in the public portal are retained by the authors and/or other copyright owners and it is a condition of accessing publications that users recognise and abide by the legal requirements associated with these rights.

- Users may download and print one copy of any publication from the public portal for the purpose of private study or research.

- You may not further distribute the material or use it for any profit-making activity or commercial gain

- You may freely distribute the URL identifying the publication in the public portal 


\title{
Fusing Simultaneous EEG and fMRI Using Functional and Anatomical Information
}

\author{
Sofie Therese Hansen*, Irene Winkler ${ }^{\dagger}$, Lars Kai Hansen*, Klaus-Robert Müller ${ }^{\dagger \ddagger}$, Sven Dähne ${ }^{\dagger}$ \\ *Department of Applied Mathematics and Computer Science, Technical University of Denmark, Kgs. Lyngby, Denmark. \\ ${ }^{\dagger}$ Department of Computer Science, Technical University of Berlin, Berlin, Germany. \\ ${ }^{\ddagger}$ Department of Brain and Cognitive Engineering, Korea University, Seoul, Republic of Korea.
}

\begin{abstract}
Simultaneously measuring electrophysical and hemodynamic signals has become more accessible in the last years and the need for modeling techniques that can fuse the modalities is growing. In this work we augment a specific fusion method, the multimodal Source Power Co-modulation (mSPoC), to not only use functional but also anatomical information. The goal is to extract correlated source components from electroencephalography (EEG) and functional magnetic resonance imaging (fMRI). Anatomical information enters our proposed extension to $\mathrm{mSPoC}$ via the forward model, which relates the activity on cortex level to the EEG sensors. The augmented $\mathrm{mSPoC}$ is shown to outperform the original version in realistic simulations where the signal to noise ratio is low or where training epochs are scarce.
\end{abstract}

\section{INTRODUCTION}

Multimodal neuroimaging techniques hold promise for enhancing our knowledge of the normal and pathological brain [1], [2]. Integrating electrophysical and hemodynamic neural responses can facilitate information not obtainable with the individual techniques [3], [4]. The electrophysical signals, e.g. magneto/electro-encephalography (M/EEG), have a high temporal resolution but low spatial resolution, whereas the hemodynamic signals, e.g. functional magnetic resonance imaging (fMRI), have low temporal resolution but high spatial resolution [5]. A technique that exploits the specific advantages of the modalities is therefore highly sought, as no gold standard is established yet. For a more exhaustive overview of multimodal analysis techniques, the interested reader is referred to [2], [6], [7]. The fusion of electrophysical and hemodynamical signals must be approached very carefully as the two categories of modalities measure different attributes of the brain activity [2], [8]. Of the many attempts to solve this problem of modeling the neurovascular coupling and combining the modalities, we will mention only a few.

In EEG source localization spatial information from fMRI can be used to guide the reconstruction [3]. This can be done in the form of a strict or soft prior on where the neural activity should be located according to the fMRI signal. This is called asymmetrical integration as one modality is given preference [8]. An example of symmetrical integration is given in [9] where Valdes-Sosa et al. describe an approach to correlate the actual generators of fMRI and EEG, i.e. the vasomotor feedforward signal and the net primary current density, respectively. This technique thus rely on both inverse temporal (of the fMRI) and inverse spatial (of the EEG) problems. These inverse problems are however very sensitive to the applied assumptions [8].

As in [9] we are interested in fusing bandpower modulations contained in the EEG with the dynamics of the fMRI signal. A recently proposed method attempts to achieve this fusion by projecting both EEG and fMRI signals into a lower dimensional source-component space. The method is called multimodal Source Power Co-modulation $(\mathrm{mSPoC})[10]$ and it is based on ideas presented in [11]. The mapping to component space is not to be confused with the previously mentioned source localization, i.e. the mapping of M/EEG recordings into a much higher dimensional cortex surface space. Specifically, $\mathrm{mSPoC}$ searches for components that exhibit maximal functional coupling between bandpower modulations (EEG component) and the hemodynamic response (fMRI component). Both modalities are given equal priority, thus making $\mathrm{mSPoC}$ a symmetrical fusion approach. Importantly, mSPoC uses only the temporal information of the modalities and is thus based on purely functional assumptions.

In this paper, we suggest to extend $\mathrm{mSPoC}$, when working with EEG and fMRI, by adding anatomical information that makes the method asymmetrical in the spatial domain. We propose to achieve this by projecting the estimated spatial activation pattern of the fMRI component to the EEG sensor space and bias the spatial activation pattern of the corresponding EEG component towards this projection. We thereby give preference to the fMRI in the spatial domain, and thus exploit the high spatial resolution of the modality.

\section{Methods}

\section{A. The Generative Model}

Let $\mathbf{X} \in \mathbb{R}^{M_{x} \times T_{x}}$ denote the EEG dataset and $\mathbf{Y} \in$ $\mathbb{R}^{M_{y} \times T_{y}}$ denote the fMRI dataset, where $M_{x / y}$ and $T_{x / y}$ denote the modality specific number of sensors and time samples, respectively. The central assumption we make is that both datasets are decomposable into what is called a set of components (or factors). The component $k$ is identified by a time course $\left(\mathbf{s}_{k}\right)$ as well as a spatial activation pattern $\left(\mathbf{a}_{k}\right)$ and may thus be regarded as a functional unit. The generative model for dataset $\mathbf{X}$ is then $\mathbf{X}=\mathbf{A}_{x} \mathbf{S}_{x}+\boldsymbol{\epsilon}_{x}$, where $\boldsymbol{\epsilon}_{x}$ is IID noise. $\mathbf{A}_{x}$ contains $K_{x}$ spatial patterns in the columns and $\mathbf{S}_{x}$ contains $K_{x}$ time courses in the rows. The same framework also holds for $\mathbf{Y}$. 


\section{B. Multimodal Source Power Co-modulation (mSPoC)}

For completeness and to facilitate the description of our expansion in section $\mathrm{II}-\mathrm{C}$ we now describe $\mathrm{mSPoC}$. For further information we refer to [10].

mSPoC seeks $K \leq \min \left(K_{x}, K_{y}\right)$ pairs of functionally coupled components in $\mathbf{X}$ and $\mathbf{Y}$. More specifically, the bandpower dynamics of a component from the EEG are assumed to co-modulate with the time course of a component in the fMRI. In the following we assume the EEG to be band-pass filtered in a frequency range of interest and epoched to match the fMRI recordings. We use the notation $\mathbf{X}(e)$ to refer to the EEG signal in epoch $e$ and $\mathbf{C}_{x x}(e)$ to denote the covariance of $\mathbf{X}(e)$ while $\mathbf{C}_{x x}$ is the covariance of the full signal $\mathbf{X}$.

In $\mathrm{mSPoC}$ the extraction of components is parametrized by means of spatial filters. The time courses of the extracted components from $\mathbf{X}$ and $\mathbf{Y}$ are given as $\hat{\mathbf{s}}_{x} \stackrel{\text { def }}{=} \mathbf{w}_{x}^{\top} \mathbf{X}$ and $\hat{\mathbf{s}}_{y} \stackrel{\text { def }}{=} \mathbf{w}_{y}^{\top} \mathbf{Y}$ for $\mathbf{w}_{x} \in \mathbb{R}^{M_{x}}$ and $\mathbf{w}_{y} \in \mathbb{R}^{M_{y}}$. Moreover the filter-pattern relation is $\hat{\mathbf{a}}_{x} \propto \mathbf{C}_{x x} \mathbf{w}_{x}$ and $\hat{\mathbf{a}}_{y} \propto \mathbf{C}_{y y} \mathbf{w}_{y}$ [12]. The bandpower dynamics of an extracted component from the EEG are given by the variance computed over the epoch $e$ and is denoted by $\phi(e)$, which is defined as $\phi(e) \stackrel{\text { def }}{=} \operatorname{Var}\left[\mathbf{w}_{x}^{\top} \mathbf{X}(e)\right]=\mathbf{w}_{x}^{\top} \mathbf{C}_{x x}(e) \mathbf{w}_{x}$.

In order to model temporal delays in the coupling between $\mathbf{X}$ and $\mathbf{Y}$, a finite-impulse-response (FIR) filter $\mathbf{w}_{\tau} \in \mathbb{R}^{N_{\tau}}$ is applied to the bandpower dynamics $\phi$. Thus, the FIR-filtered bandpower dynamics are defined as

$$
\begin{aligned}
h(\phi)(e) & \stackrel{\text { def }}{=} \sum_{i=0}^{N_{\tau}-1} \mathbf{w}_{\tau}(i+1) \cdot \phi(e-i) \\
& =\mathbf{w}_{x}^{\top} \underbrace{\left(\sum_{i=0}^{N_{\tau}-1} \mathbf{w}_{\tau}(i+1) \cdot \mathbf{C}_{x x}(e-i)\right)}_{\stackrel{\text { def }}{=} \mathbf{C}_{h}(e)} \mathbf{w}_{x},(2)
\end{aligned}
$$

Note that $h(\phi)(e)$ depends on $\mathbf{w}_{x}$ as well as on $\mathbf{w}_{\tau}$.

With these definitions, the $\mathrm{mSPoC}$ objective function for functional coupling between $\mathbf{X}$ and $\mathbf{Y}$ is given by

$$
\begin{aligned}
& \max _{\mathbf{w}_{x}, \mathbf{w}_{\tau}, \mathbf{w}_{y}} \operatorname{Cov}\left(h(\boldsymbol{\phi}), \hat{\mathbf{s}}_{y}\right) \\
& \text { s.t. } \operatorname{Var}\left(\hat{\mathbf{s}}_{x}\right)=\operatorname{Var}(h(\phi))=\operatorname{Var}\left(\hat{\mathbf{s}}_{y}\right)=1 .
\end{aligned}
$$

Note that if $\mathbf{w}_{x}$ is provided, $\phi$ can be computed and the optimization problem (now only for $\mathbf{w}_{\tau}$ and $\mathbf{w}_{y}$ ) reduces to the well known canonical correlation analysis (CCA) with temporal embedding of $\phi$ [13]. If, on the other hand, $\mathbf{w}_{\tau}$ and $\mathbf{w}_{y}$ are provided, then $\mathbf{C}_{h}(e)$ and $\hat{\mathbf{s}}_{y}$ can be computed and the resulting optimization problem (now only for $\mathbf{w}_{x}$ ) leads to the following generalized eigenvalue problem

$$
\mathbf{C}_{z} \mathbf{w}_{x}=\lambda \mathbf{C}_{x x} \mathbf{w}_{x}
$$

where $\mathbf{C}_{z} \stackrel{\text { def }}{=} \sum_{e} \mathbf{C}_{h}(e) \cdot \hat{\mathbf{s}}_{y}(e)$.

The $\mathrm{mSPoC}$ objective is optimized by iterating over:

$0)$ Initialize $\mathbf{w}_{x}$ randomly.

1) Given $\mathbf{w}_{x}$, solve for $\mathbf{w}_{\tau}$ and $\mathbf{w}_{y}$ using CCA.

2) Given $\mathbf{w}_{\tau}$ and $\mathbf{w}_{y}$, solve for $\mathbf{w}_{x}$ using Eq. (4).

3) Repeat steps 1 and 2 until convergence.

\section{C. $m S P o C$ With Anatomical Information: $\mathrm{mSPoC}_{\gamma} / \mathrm{mSPoC}_{\vec{\gamma}}$}

We now include the assumption that the extracted components should not only be functionally linked but also anatomically related. Thus we assume the spatial activation pattern of the EEG component, denoted by $\hat{\mathbf{a}}_{x}$, to be similar to the activation pattern of the corresponding fMRI component, denoted by $\hat{\mathbf{a}}_{y}$. In order to relate $\hat{\mathbf{a}}_{x}$ to $\hat{\mathbf{a}}_{y}$, we project $\hat{\mathbf{a}}_{y}$ to the EEG sensor space using a lead field matrix $\mathbf{L} \in \mathbb{R}^{M_{x} \times M_{y}}$; i.e. $\stackrel{y \rightarrow}{\mathbf{a}_{x}} \stackrel{\text { def }}{=} \mathbf{L} \hat{\mathbf{a}}_{y}$ is the projected fMRI pattern.

Finding the $\mathbf{w}_{x}$ that optimally extracts the temporal activity from the EEG given a spatial pattern, in our case ${ }_{\mathbf{a}} \vec{a}_{x}$, can be achieved by the linearly constraint minimum variance (LCMV) beamformer [14]. In the LCMV approach, the optimal $\mathbf{w}_{x}$ is derived by minimizing $\mathbf{w}_{x}^{\top} \mathbf{C}_{x x} \mathbf{w}_{x}=\operatorname{Var}\left(\hat{\mathbf{s}}_{x}\right)$, subject to the constraint $\mathbf{w}_{x}^{\top} \mathbf{a}_{x}=1$. Here we find it convenient to modify [14] to instead maximize $\left(\mathbf{w}_{x}^{\top y} \mathbf{a}_{x}\right)^{2}$, corresponding to

$$
\max _{w_{x}} \mathbf{w}_{x}^{\top} \mathbf{C}_{a a} \mathbf{w}_{x} \text { s.t. } \operatorname{Var}\left(\hat{\mathbf{s}}_{x}\right)=1,
$$

where $\mathbf{C}_{a a} \stackrel{\text { def }}{=} y \mathbf{a}_{x} \mathbf{a}_{x}^{\top}$ is a rank-one matrix. Note that the solution to this problem is (up to a scaling) identical to the one of the original LCMV, both satisfying $\mathbf{w}_{x} \propto \mathbf{C}_{x x}^{-1} \mathbf{a}_{x}$.

These insights now allow us to integrate the anatomical information contained in the lead field matrix $\mathbf{L}$ into the $\mathrm{mSPoC}$ objective. Specifically, we replace Eq. (4) by

$$
\left.\left((1-\gamma) \mathbf{C}_{z}+\gamma \mathbf{C}_{a a}\right)\right) \mathbf{w}_{x}=\lambda \mathbf{C}_{x x} \mathbf{w}_{x}
$$

where $\gamma \in[0,1]$ allows to smoothly interpolate between using only functional information $(\gamma=0$, corresponding to the original $\mathrm{mSPoC}$ ) and using only anatomical information $(\gamma=1)$. Thus $\gamma$ is the strength of the spatial constraint. We refer to this version of $\mathrm{mSPoC}$ as $\mathrm{mSPoC}_{\gamma}$.

As a further variant, we propose to threshold the estimate of $\hat{\mathbf{a}}_{y}$ prior to the computation of $\mathbf{a}_{x}$. Specifically, we propose to set all values in $\hat{\mathbf{a}}_{y}$ to zero, except for the maximum magnitude value. Thereby, we reduce the influence of noise while at the same time dealing with a known weakness of beamformers, namely a performance decrease when the spatial extent of the source increases [15]. This version of $\mathrm{mSPoC}$ will be referred to as $\mathrm{mSPoC}_{\bar{\gamma}}$.

\section{Simulated Data}

The simulated EEG signal has 70 electrodes and the fMRI activity is assumed to be projected to the cortical surface consisting of 8196 vertices. The two modalities are set to have one source component in common. In addition to sharing temporal dynamics the component in the two modalities also share spatial patterns. The spatial pattern of the EEG component is thus the projection of the spatial pattern of the fMRI component through a lead field matrix. The lead field matrix was generated in SPM8 [16] using the boundary element method (BEM). Here a structural MRI scan of a subject was used; dataset was provided by Henson and Wakeman [3].

The generated EEG signal has frequencies between 8 and $12 \mathrm{~Hz}$ and has a sampling frequency of $200 \mathrm{~Hz}$. The sampling 

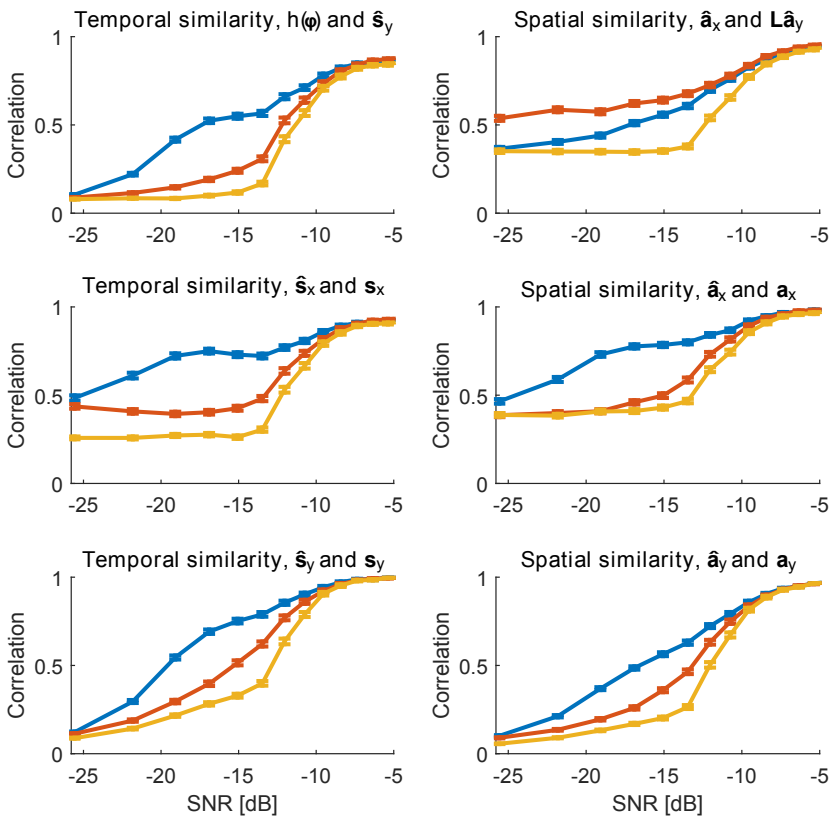

a) Influence of the SNR
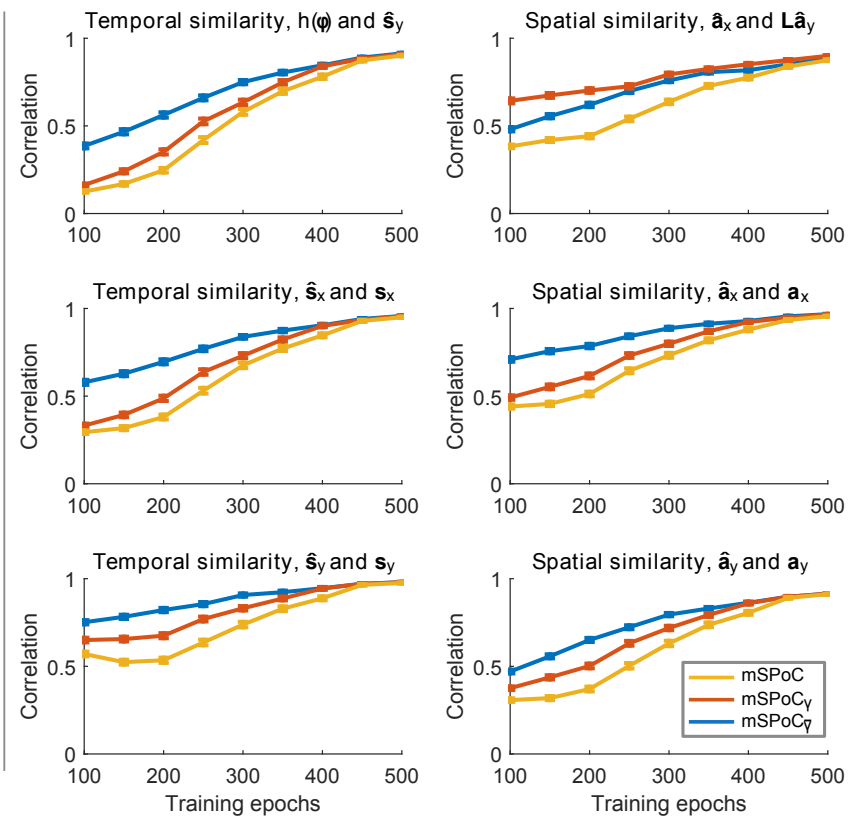

b) Influence of the number of training epochs

Fig. 1. Correlations obtained using the original and augmented mSPoCs. a) Training was performed on 250 epochs $(\sim 4$ min) on data with a varying SNR. b) Training was performed on a varying number of epochs on data with $\mathrm{SNR}=-12 \mathrm{~dB}$. The time course correlation is reported on a held out test set of 600 epochs (10 min data). Patterns are calculated on the training data and $\mathbf{L}$ is the lead field matrix. We show the mean of 500 repetitions together with the standard error on the mean. $\mathrm{mSPoC}_{\gamma}$ and $\mathrm{mSPoC}_{\bar{\gamma}}$ include crossvalidation to estimate $\gamma$.

frequency of fMRI is $1 \mathrm{~Hz}$, meaning that the EEG is divided into epochs of 200 samples each. The fMRI component and the envelope of the paired EEG component have a frequency of maximum $1 / 4 \mathrm{~Hz}$, i.e the coupled activity oscillates with $1 / 4 \mathrm{~Hz}$ or below. The fMRI signal is temporally delayed using a Gaussian shaped hemodynamic response function (HRF) with a length of 15 seconds $\left(N_{\tau}=15\right)$.

The EEG and fMRI additionally have 14 and 499, respectively, background source components. The spatial pattern of each source component is equivalent to a delimited Gaussian at the cortex level. The simulated EEG signal is a weighted sum over the Frobenius normalized source and background activity; i.e. $\mathbf{X}=\lambda \mathbf{X}_{\text {source }} /\left\|\mathbf{X}_{\text {source }}\right\|_{F}+(1-$ $\lambda) \mathbf{X}_{\text {background }} /\left\|\mathbf{X}_{\text {background }}\right\|_{F}$. The SNR is here defined as $20 \log 10(\lambda /(1-\lambda))$. Same structure applies for the fMRI.

We vary both the SNR and the number of epochs available for training, and test the temporal correlation on 600 epochs. To estimate the level of anatomical information needed $(\gamma)$ in $\mathrm{mSPoC}_{\gamma}$ and $\mathrm{mSPoC}_{\bar{\gamma}}$ we perform five-fold crossvalidation on the training data with $\gamma=(0, \ldots, 1)$ in 25 steps.

\section{RESULTS}

In Figure 1 we show the performance of the original $\mathrm{mSPoC}$ and the two augmented versions by their dependence on a) the SNR and b) the number of training epochs. The first row of Figure 1 shows the intermodal temporal and spatial similarity, i.e. the correlation between the estimated EEG and fMRI component. The middle row shows the temporal and spatial similarity of the true and estimated EEG component.
The same is shown for fMRI in the bottom row. In real data applications we would only be able to show the top row.

As seen in Figure 11 $) \mathrm{mSPoC}_{\gamma}$ and especially $\mathrm{mSPoC}_{\bar{\gamma}}$ outperform the original $\mathrm{mSPoC}$ for most SNR levels. For higher SNRs $(>-10 \mathrm{~dB})$ the three methods converge to the same performance. It is evident from the middle and bottom rows of Figure $1 \mathrm{a}$ ) that the augmentation improves the recovery of the true components both on the EEG side and on the fMRI side.

Superiority of $\mathrm{mSPoC}_{\gamma}$ compared to $\mathrm{mSPoC}_{\bar{\gamma}}$ is only found on the correlation of the estimated EEG pattern and the projected estimated fMRI pattern. This appears to be an overfitting of the EEG pattern to the noise components in the estimated fMRI pattern, as the spatial correlations of the estimated patterns of the two modalities to the true pattens do not show the same degree of improvement.

Analyzing Figure 1p) shows the same trend as Figure 19), only now for the number of training epochs. The temporal correlation of the bandpowered EEG and the fMRI signal is highest using $\mathrm{mSPoC}_{\bar{\gamma}}$ followed by $\mathrm{mSPoC}_{\gamma}$. More specifically $\mathrm{mSPoC}_{\bar{\gamma}}$ outperforms $\mathrm{mSPoC}$ when fewer than 400 epochs (here corresponding to $6.7 \mathrm{~min}$ ) are available for training.

We investigate $\mathrm{mSPoC}_{\bar{\gamma}}$ further in Figure 2 where we explore the influence on the temporal correlation of the SNR and the strength of the spatial constraint $(\gamma)$. We see that when having low SNR mSPoC benefits from anatomical information and the biggest improvement can be found here compared to $\mathrm{mSPoC}$ (which corresponds to $\gamma=0$ ). However, for higher levels of SNR relying too much on anatomical information 


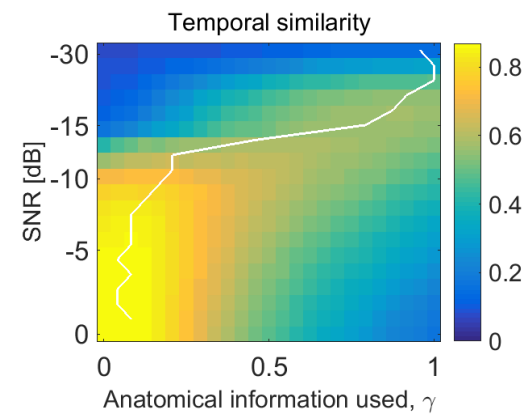

Fig. 2. For $\mathrm{mSPoC}_{\bar{\gamma}}$ : The influence of $\gamma$ and the SNR on the correlation between the temporally convolved EEG component and the fMRI component, i.e. $h(\phi)$ and $\hat{\mathbf{s}}_{y}$. A $\gamma=0$ corresponds to the original mSPoC. The mean test correlation of 600 epochs across 500 data sets each trained on 250 epochs is shown. The white line indicates the optimum strength of the spatial constraint

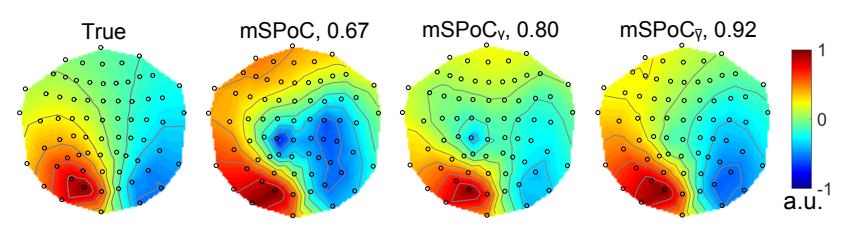

Fig. 3. An example of the estimated EEG patterns, where SNR $=-12 \mathrm{~dB}$ and training is performed on 250 epochs $(\sim 4 \mathrm{~min})$. The correlations between the true and estimated patterns are shown.

worsen the solution and the spatial constraint should thus be small here.

An example of the estimated EEG patterns found by the three $\mathrm{mSPoC}$ versions is shown in Figure 3 together with the true pattern. The scalp map found with $\mathrm{mSPoC}_{\bar{\gamma}}$ has highest correspondence with the true scalp map.

\section{Discussion AND CONCLUSION}

The original multimodal Source Power Co-modulation $(\mathrm{mSPoC})$ was in [10] compared to CCA where it was found to be superior. In this work we have introduced two expansions of $\mathrm{mSPoC}$ that exploit anatomical information. Both improve the extraction of correlated EEG and fMRI components. However, $\mathrm{mSPoC}_{\bar{\gamma}}$ demonstrates higher potential for future use as it seems to avoid overfitting to noise components.

The next steps include investigating how $\mathrm{mSPoC}_{\bar{\gamma}}$ reacts when certain assumptions are violated. We e.g. need to explore what happens when the paired components which are temporally correlated do not match spatially. The accuracy of the lead field matrix's projection of the fMRI to the EEG sensors could also be expected to influence the solution. The method should also be applied to real EEG and fMRI data to verify its applicability in multimodal neuroimaging. In the simulations we assumed that the fMRI activity was projected to the cortex. This projection can be performed with real data using a Voronoï interpolation scheme [17] or using SPM8 [16].

The preliminary results hold promise to improve the extraction of correlated pairs in situations where the SNR is low and/or where few training epochs are available. The method could thus potentially be used to investigate the correlation of the modalities as a function of time.

\section{ACKNOWLEDGMENT}

LKH acknowledges support from the Danish Lundbeck Foundation via the Center for Integrated Molecular Brain Imaging (CIMBI). SD acknowledges funding by the German Research Foundation (DFG) grant no. MU 987/19-1 and support by the Bernstein Center for Computational Neuroscience, Berlin through the graduate program GRK 1589/1. KRM acknowledges support by the Brain Korea 21 Plus Program through the National Research Foundation of Korea funded by the Ministry of Education.

\section{REFERENCES}

[1] N. D. Schiff, "Multimodal neuroimaging approaches to disorders of consciousness," The Journal of head trauma rehabilitation, vol. 21, no. 5, pp. 388-397, 2006.

[2] J. Sui, T. Adali, Q. Yu, J. Chen, and V. D. Calhoun, "A review of multivariate methods for multimodal fusion of brain imaging data," Journal of neuroscience methods, vol. 204, no. 1, pp. 68-81, 2012.

[3] R. N. Henson, D. G. Wakeman, V. Litvak, and K. J. Friston, "A Parametric Empirical Bayesian Framework for the EEG/MEG Inverse Problem: Generative Models for Multi-Subject and Multi-Modal Integration." Frontiers in human neuroscience, vol. 5, p. 76, 2011.

[4] S. M. Plis, V. D. Calhoun, M. P. Weisend, T. Eichele, and T. Lane, "MEG and fMRI Fusion for Non-Linear Estimation of Neural and BOLD Signal Changes." Frontiers in neuroinformatics, vol. 4, p. 114, 2010.

[5] S. Baillet, J. C. Mosher, and R. M. Leahy, "Electromagnetic brain mapping," Signal Processing Magazine, IEEE, vol. 18, no. 6, pp. 14-30, 2001.

[6] F. Bießmann, S. M. Plis, F. C. Meinecke, T. Eichele, and K.-R. Müller, "Analysis of multimodal neuroimaging data," IEEE Reviews in Biomedical Engineering, vol. 4, pp. 26-58, 2011.

[7] S. Dähne, F. Bießman, W. Samek, S. Haufe, D. Goltz, C. Gundlach, A. Villringer, S. Fazli, and K.-R. Müller, "Multivariate machine learning methods for fusing functional multimodal neuroimaging data," Proceedings of the IEEE, 2015, accepted.

[8] X. Lei, P. A. Valdes-Sosa, and D. Yao, "EEG/fMRI fusion based on independent component analysis: integration of data-driven and modeldriven methods." Journal of integrative neuroscience, vol. 11, no. 3, pp. 313-337, 2012.

[9] P. A. Valdes-Sosa, J. M. Sanchez-Bornot, R. C. Sotero, Y. IturriaMedina, Y. Aleman-Gomez, J. Bosch-Bayard, F. Carbonell, and T. Ozaki, "Model driven EEG/fMRI fusion of brain oscillations." Human brain mapping, vol. 30, no. 9, pp. 2701-2721, 2009.

[10] S. Dähne, F. Bießmann, F. C. Meinecke, J. Mehnert, S. Fazli, and K.R. Müller, "Integration of multivariate data streams with bandpower signals," IEEE Transactions on Multimedia, vol. 15, no. 5, pp. 10011013, 2013.

[11] S. Dähne, F. C. Meinecke, S. Haufe, J. Höhne, M. Tangermann, K.-R. Müller, and V. V. Nikulin, "SPoC: a novel framework for relating the amplitude of neuronal oscillations to behaviorally relevant parameters," NeuroImage, vol. 86, no. 0, pp. 111-122, 2014.

[12] S. Haufe, F. Meinecke, K. Görgen, S. Dähne, J.-D. Haynes, B. Blankertz, and F. Bießmann, "On the interpretation of weight vectors of linear models in multivariate neuroimaging," NeuroImage, vol. 87, pp. 96-110, 2014.

[13] F. Bießmann, F. C. Meinecke, A. Gretton, A. Rauch, G. Rainer, N. K. Logothetis, and K.-R. Müller, "Temporal kernel cca and its application in multimodal neuronal data analysis," Machine Learning, vol. 79, no. 1-2, pp. 5-27, 2010.

[14] B. D. van Veen and K. M. Buckley, "Beamforming: A versatile approach to spatial filtering," IEEE ASSP Magazine, vol. 5, pp. 4-24.

[15] M. J. Brookes, C. M. Stevenson, G. R. Barnes, A. Hillebrand, M. I. G. Simpson, S. T. Francis, and P. G. Morris, "Beamformer reconstruction of correlated sources using a modified source model," NeuroImage, vol. 34, no. 4, pp. 1454-1465.

[16] J. Ashburner, C.-C. Chen, R. Moran, R. N. Henson, V. Glauche, and C. Phillips, "SPM8 manual," The FIL Methods Group, Tech. Rep., 2012.

[17] C. Grova, S. Makni, G. Flandin, P. Ciuciu, J. Gotman, and J. B. Poline, "Anatomically informed interpolation of fMRI data on the cortical surface." NeuroImage, vol. 31, no. 4, pp. 1475-86, 2006. 